\title{
Robustness Analysis with Mixed Performance Objectives ${ }^{1}$
}

\author{
Juanyu Bu Mario Sznaier ${ }^{2}$ \\ Department of Electrical Engineering \\ The Pennsylvania State University \\ University Park, PA 16802 \\ msznaier@frodo.ee.psu.edu
}

\begin{abstract}
Mixed performance control problems have been the object of much attention lately. These problems allow for capturing different performance specifications without resorting to approximations or the use of weighting functions. However, up to date most of the work concerning multiobjective control is limited to guaranteeing nominal performance and robust stability. In this paper we analyze robust performance for a class of mixed problems. The main results of the paper furnish sufficient conditions for guaranteeing performance (in the $\ell_{\infty}$ sense) under model perturbations having an $\ell_{2}$ to $\ell_{2}$ bounded norm. These condition can be combined with previously proposed multiobjective control synthesis techniques to obtain controllers guaranteeing robust performance.
\end{abstract}

\section{Introduction}

Multiobjective control problems have been the object of much attention lately (see [18] for references on recent work on multiobjective control). In particular, $\mathcal{H}_{2} / \mathcal{H}_{\infty}$ mixed control has been extensively investigated since its introduction (see for instance $[2,7,10,11]$ and references therein). More recently $\ell^{1} / \mathcal{H}_{\infty}[21,17]$ and $\ell^{1} / \mathcal{H}_{2}$ control problems have been formulated [20]. Given the difficulty in addressing multiple performance objectives, these papers concentrate on nominal performance / robust stability type designs, i.e. controllers that guarantee stability for a family of plants, while at the same time, achieving optimal performance

'This work was supported in part by NSF under grants ECS-9211169 and ECS-9625920

${ }^{2}$ Corresponding author. for the nominal plant.

However, even if the closed-loop system is guaranteed to remain stable for off-nominal conditions, performance can be severely degraded. This has led to a recent research effort geared towards synthesizing controllers achieving robust performance. Most of this work concentrates on developing analysis tools to assess worst-case performance in the presence of norm-bound model uncertainty, although some partial synthesis results are also available $([14,13])$. Moreover, a large portion of this research effort has been directed towards analyzing performance when the exogenous input is a known, given signal. Recent work in this respect includes $[14,4,5,1]$ (see also [8] for the related problem of robust steady-state tracking).

Following along these lines, in this paper we study the problem of robust performance (in the $\ell^{\infty}$ sense) to a fixed input, when the system is subject to model uncertainty with bounded $\ell^{2}$ induced norm. This problem can be though of as a natural extension of the mixed $\ell^{\infty} / \mathcal{H}_{\infty}$ problem formulated in $[15,16]$. The main result of the paper provides sufficient conditions for achieving robust performance under these conditions. One of these conditions, given in terms of the spectral radius of a matrix, provides a simple robustness check. The second condition reduces conservatism, at the price of more involved computations. These results are illustrated with a simple example.

\section{Notation and preliminary results}

\subsection{Notation}

Given a vector $x \in R^{n}$ its 2 -norm is defined as $\|x\|_{2} \triangleq \sum_{i=0}^{n} x_{i}^{2}$ and its infinity norm as 
$\|x\|_{\infty} \triangleq \max _{i}\left|x_{i}\right| . \quad \ell^{2}$ denotes the space of bounded energy sequences $h=\left\{h_{i}\right\}$ with the norm $\|h\|_{l^{2}} \triangleq\left(\sum_{i=0}^{\infty}\left|h_{i}\right|^{2}\right)^{\frac{1}{2}}<\infty . \ell^{\infty}$ denotes the linear space of bounded sequences equipped with the norm $\|h\|_{\ell \infty} \triangleq \sup _{i \geq 0}\left|h_{i}\right|<\infty$.

$\mathcal{H}_{\infty}$ denotes the space of complex valued matrix functions that are analytic outside the unit disk. The norm on $\mathcal{H}_{\infty}$ is defined by $\|G(z)\|_{\mathcal{H}_{\infty}} \triangleq e s s \sup _{|z|>1} \bar{\sigma}(G(z))$, where $\bar{\sigma}$ denotes the largest singular value.

Assume now that $H: \ell^{2} \rightarrow \ell^{2}$ is a bounded linear operator defined by the usual convolution relation $y=H * u$. Its $\ell^{2}$ induced norm is defined as $\|H\|_{\ell^{2} \rightarrow \ell^{2}} \doteq \sup _{\|u\|_{\ell^{3} \leq 1}}\|H * u\|_{\ell^{2}}$. It is well known that if $H$ is linear time-invariant, $\|H\|_{\ell^{2} \rightarrow \ell^{2}}=\|H\|_{\mathcal{H}_{\infty}}$.

In the sequel, we shall assume that the system uncertainty block $\Delta$ belongs to the following class:

$$
\mathbf{D}_{2} \triangleq\left\{\Delta: \Delta \text { is causal and }\|\Delta\|_{\ell^{2} \rightarrow \ell^{2}}<1\right\}
$$

\subsection{The robust mixed $\ell^{\infty} / \mathcal{H}_{\infty}$ problem}

Definition 2.1 Given the input $w \in \ell^{\infty}$, the system shown in Figure 1 achieves robust performance in the mixed $\ell^{\infty} / \mathcal{H}_{\infty}$ sense if the interconnection is robustly stable and such that $\|z\|_{\ell^{\infty}} \leq 1$ for all $\Delta,\|\Delta\|_{\ell^{2} \rightarrow \ell^{2}}<1$.

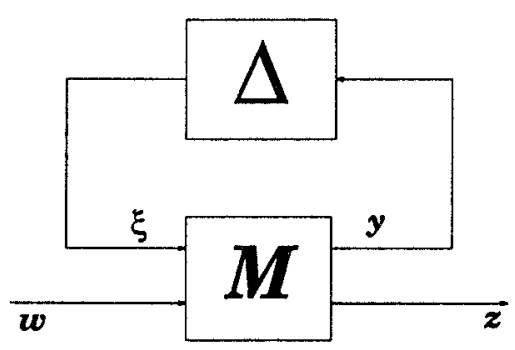

Figure 1: Robust mixed $\ell^{\infty} / \mathcal{H}_{\infty}$ problem

In the sequel we derive several conditions guaranteeing mixed $\ell^{\infty} / \mathcal{H}_{\infty}$ robust performance. To this effect we begin by introducing the following two lemmas about non-negative matrices.

Lemma 2.1 Let $A$ be a square non-negative matrix, i.e., $a_{i j} \geq 0$. Then $\rho(A) \leq 1$ if and only if the inequalities $x<A x$ and $x \geq 0$ have no solution, where the vector inequalities should be interpreted in a componentwise sense.

Proof: See, for instance, References $[3,6]$.

Lemma 2.2 Let $A$ be a non-negative matrix partitioned as $\left(\begin{array}{ll}A_{11} & A_{12} \\ A_{21} & A_{22}\end{array}\right)$, and suppose $\rho\left(A_{22}\right)<1$. Then $\rho(A) \leq 1$ if and only if $\rho\left(A_{11}+A_{12}\left(I-A_{22}\right)^{-1} A_{21}\right) \leq 1$.

Proof: The proof, omitted for space reasons, follows from Lemma 2.1 and standard properties of non negative matrices.

\section{Robust mixed $\ell^{\infty} / \mathcal{H}_{\infty}$ performance analysis}

In this section we provide some conditions guaranteering robust performance (in the $\ell^{\infty}$ sense) against one-block (unstructured), LTV perturbations having bounded $\ell^{2} \rightarrow \ell^{2}$ induced norm.

Theorem 3.1 Assume that $\left\|M_{11}\right\|_{\mathcal{H}_{\infty}} \leq 1$. If

$$
\rho\left(\begin{array}{ll}
\left\|M_{11}\right\| \mathcal{H}_{\infty} & \left\|M_{12} w\right\|_{\ell^{2}} \\
\left\|M_{21}\right\|_{\mathcal{H}_{2}} & \left\|M_{22} w\right\|_{\ell^{\infty}}
\end{array}\right) \leq 1
$$

then the system $M$ has mixed robust performance for all $L T V$ perturbations $\Delta \in \mathbf{D}_{\mathbf{2}}$.

Proof: It is sufficient to show that

$$
\begin{aligned}
& \sup _{\Delta \in \mathrm{D}_{2}}\|z\|_{\ell_{\infty}}>1 \Longrightarrow \\
& \rho\left(\begin{array}{cc}
\left\|M_{11}\right\|_{\mathcal{H}_{\infty}} & \left\|M_{12} w\right\|_{\ell^{2}} \\
\left\|M_{21}\right\|_{\mathcal{H}_{2}} & \left\|M_{22} w\right\|_{\ell^{\infty}}
\end{array}\right)>1
\end{aligned}
$$

Suppose for some $\Delta \in \mathbf{D}_{\mathbf{2}},\|z\|_{\ell \infty}>1$. For this $\Delta$, it follows that

$$
\left(\begin{array}{l}
y \\
z
\end{array}\right)=\left(\begin{array}{ll}
M_{11} & M_{12} \\
M_{21} & M_{22}
\end{array}\right)\left(\begin{array}{c}
\xi \\
w
\end{array}\right)
$$

It is well known that $\sup _{\|x\|_{L^{2} \leq 1}}\|M x\|_{\ell^{\infty}}=\|M\|_{\mathcal{H}_{2}}$. and $\sup _{\|x\|_{\ell^{2} \leq 1} \leq 1}\|M x\|_{\ell^{2}}=\|M\|_{\mathcal{H}_{\infty}}$. Thus, from the triangle inequality, it follows that

$$
\left(\begin{array}{c}
\|y\|_{\ell^{2}} \\
\|z\|_{\ell^{\infty}}
\end{array}\right)=\left(\begin{array}{ll}
\left\|M_{11}\right\|_{\mathcal{H}_{\infty}} & \left\|M_{12} w\right\|_{\ell^{2}} \\
\left\|M_{21}\right\|_{\mathcal{H}_{2}} & \left\|M_{22} w\right\|_{\ell^{\infty}}
\end{array}\right)\left(\begin{array}{c}
\|\xi\|_{\ell^{2}} \\
1
\end{array}\right)
$$

Since $\Delta \in \mathbf{D}_{\mathbf{2}}$ and $\|z\|_{\ell \infty}>1$, it also follows that $\|\xi\|_{\ell^{2}}<\|y\|_{\ell^{2}}$ and

$$
\left(\begin{array}{c}
\|\xi\|_{l^{2}} \\
1
\end{array}\right)<\left(\begin{array}{c}
\|y\|_{\ell^{2}} \\
\|z\|_{\ell^{\infty}}
\end{array}\right)
$$


Therefore,

$$
\left(\begin{array}{c}
\|\xi\|_{\ell^{2}} \\
1
\end{array}\right)<\left(\begin{array}{cc}
\left\|M_{11}\right\|_{\mathcal{H}_{\infty}} & \left\|M_{12} w\right\|_{\ell^{2}} \\
\left\|M_{21}\right\|_{\mathcal{H}_{2}} & \left\|M_{22} w\right\|_{\ell^{2}}
\end{array}\right)\left(\begin{array}{c}
\|\xi\|_{\ell^{2}} \\
1
\end{array}\right)
$$

By Lemma 2.1 this implies

$$
\rho\left(\begin{array}{ll}
\left\|M_{11}\right\|_{\mathcal{H}_{\infty}} & \left\|M_{12} w\right\|_{\ell^{2}} \\
\left\|M_{21}\right\|_{\mathcal{H}_{2}} & \left\|M_{22} w\right\|_{\ell^{\infty}}
\end{array}\right)>1
$$

Corollary 3.2 If $\left\|M_{11}\right\|_{\mathcal{H}_{\infty}}<1$, then for $a$ fixed input $w$ we have:

$$
\begin{aligned}
& \sup _{\Delta \in \mathrm{D}_{2}}\|z\|_{\ell \infty} \leq\left\|M_{22} w\right\|_{\ell \infty} \\
+ & \mid M_{21}\left\|\mathcal{H}_{2}\left(1-\left\|M_{11}\right\|_{\mathcal{H}_{\infty}}\right)^{-1}\right\| M_{12} w \|_{\ell 2}
\end{aligned}
$$

Next we obtain a less conservative condition, at the price of increased computational complexity. Begin by considering the worst case value of $\|y\|_{2}=\left\|M_{11} \xi+M_{12} w\right\|_{2}$ when the input $\xi$ is constrained to the $\gamma$-ball in $\ell^{2}$.

Lemma 3.1 Let $G_{\gamma}(w) \triangleq \sup _{\|\xi\|_{\ell^{2}} \leq \gamma} \| M_{11 \xi}+$ $M_{12} w \|_{L^{2}}^{2}$. Then the following properties hold:

1. $G_{\gamma}(w)$ is a continuous, monotonically increasing, and concave function of $\gamma$ for $\gamma \geq 0$.

2. Suppose $\left\|M_{11}\right\|_{\mathcal{H}_{\infty}}<1$. Then the equation

$$
G_{\gamma}(w)=\gamma
$$

has a unique solution $\gamma^{*}$. Moreover $\gamma^{*}$ satisfies

$$
\frac{\left\|M_{12} w\right\|_{\ell^{2}}^{2}}{1-\left\|M_{11}\right\|_{\mathcal{H}_{\infty}}^{2}} \leq \gamma^{*} \leq \frac{\left\|M_{12} w\right\|_{\ell^{2}}^{2}}{\left(1-\left\|M_{11}\right\|_{\mathcal{H}_{\infty}}\right)^{2}}
$$

Proof: omitted for space reasons.

Similarly, we consider now worst-case performance in the $\ell^{\infty}$ sense. Define

$$
F_{\gamma}(w)=\sup _{\|\xi\|_{\ell^{2} \leq \gamma}}\left\|M_{21} \xi+M_{22} w\right\|_{\ell^{\infty}}
$$

Let $z=M_{21} \xi+M_{22} w$. Clearly for each $N \geq 0$,

$$
\begin{aligned}
& |z(N)|=\left|\psi(N)+\sum_{i=0}^{N} m(i) \xi(N-i)\right| \leq \\
& |\psi(N)|+\left|\sum_{i=0}^{N} m(i) \xi(N-i)\right|
\end{aligned}
$$

$$
\begin{aligned}
& \leq|\psi(N)|+\sqrt{\sum_{i=0}^{N} m^{2}(i)} \sqrt{\sum_{i=0}^{N} \xi^{2}(i)} \leq \\
& |\psi(N)|+\gamma \sqrt{\sum_{i=0}^{N} m^{2}(i)}
\end{aligned}
$$

where $\{m(i)\}$ denotes the impulse response of $M_{21}$ and where $\psi=M_{22} w$. By choosing $\xi$ as:

$\xi(i)=\left\{\begin{array}{l}\operatorname{sign}\left[\left(M_{22} w\right)(N)\right] \frac{\gamma}{\sqrt{\sum_{i=0}^{N} m^{2}(i)}} m(N-i), \\ i=0,1, \cdots, N \\ 0 \text { elsewhere }\end{array}\right.$

we have that $\|\xi\|_{\ell^{2}}=\gamma$ and

$$
|z(N)|=|\psi(N)|+\gamma \sqrt{\sum_{i=0}^{N} m^{2}(i)}
$$

This implies that

$$
F_{\gamma}(w)=\sup _{N}\left\{|\psi(N)|+\gamma \sqrt{\sum_{i=0}^{N} m^{2}(i)}\right\}
$$

If the supreme is achieved for some finite $N=$ $N(\gamma)$, it follows that

$$
F_{\gamma}(w)=|\psi(N)|+\gamma \sqrt{\sum_{i=0}^{N} m^{2}(i)} \triangleq b_{\gamma}+a_{\gamma} \gamma
$$

On the other hand, if

$$
\begin{gathered}
F_{\gamma}(w)=\lim _{N \rightarrow \infty}\left\{|\psi(N)|+\gamma \sqrt{\sum_{i=0}^{N} m^{2}(i)}\right\} \\
=\lim _{N \rightarrow \infty}|\psi(N)|+\gamma \lim _{N \rightarrow \infty} \sqrt{\sum_{i=0}^{N} m^{2}(i)} \triangleq b_{\gamma}+a_{\gamma} \gamma
\end{gathered}
$$

where $a_{\gamma}=\left\|M_{21}\right\|_{\ell^{2}}$ and $b_{\gamma}=\lim _{N \rightarrow \infty}|\psi(N)|$. Therefore, it is always possible to write:

$$
\begin{array}{ll}
F_{\gamma}(w)=a_{\gamma} \gamma+b_{\gamma}, & 0 \leq a_{\gamma} \leq\left\|M_{21}\right\|_{\ell 2} \\
& 0 \leq b_{\gamma} \leq\|\psi\|_{\ell \infty} .
\end{array}
$$

Morevoer, from the definition of $F_{\gamma}$ it follows immediately that for any $\gamma_{0} \neq \gamma$,

$$
F_{\gamma}(w) \geq a_{\gamma} \gamma_{0}+b_{\gamma}
$$

Lemma 3.2 The function $F_{\gamma}(w)$ has the following properties: 
1. $F_{\gamma}(w)$ is a continuous, monotonically non-decreasing, convex function of $\gamma$ for $\gamma \geq 0$.

2. For any $\gamma_{1}, \gamma_{2} \geq 0$,

$$
\left|F_{\gamma_{1}}(w)-F_{\gamma_{2}}(w)\right| \leq\left\|M_{21}\right\|_{\ell^{2}}\left|\gamma_{1}-\gamma_{2}\right|
$$

Proof: Monotonicity follows immediately from the definition of $F_{\gamma}$. To prove item 2, given $\gamma_{1}, \gamma_{2} \geq 0$, let $\gamma_{3}=\lambda \gamma_{1}+(1-\lambda) \gamma_{2}, 0 \leq \lambda \leq 1$. It follows from (8) and (9) that

$$
\begin{aligned}
F_{\gamma_{3}}(w) & =a_{\gamma_{3}} \gamma_{3}+b_{\gamma_{3}}=\lambda\left(a_{\gamma_{3}} \gamma_{1}+b_{\gamma_{3}}\right) \\
& +(1-\lambda)\left(a_{\gamma_{3}} \gamma_{2}+b_{\gamma_{3}}\right) \\
& \leq \lambda F_{\gamma_{1}}(w)+(1-\lambda) F_{\gamma_{2}}(w)
\end{aligned}
$$

Therefore, $F_{\gamma}(w)$ is a convex function of $\gamma \geq 0$. Moreover, for any $\gamma_{1} \geq \gamma_{2} \geq 0$,

$$
\begin{aligned}
\mathrm{F}_{\gamma_{1}}(\mathrm{w})-\mathrm{F}_{\gamma_{2}}(\mathrm{w}) & \leq a_{\gamma_{1}}\left(\gamma_{1}-\gamma_{2}\right) \\
\leq & \leq M_{21} \|_{\ell^{2}}\left(\gamma_{1}-\gamma_{2}\right)
\end{aligned}
$$

Now, we are ready to present the main result of this section, providing a less conservative condition for mixed $\ell^{\infty} / \mathcal{H}_{\infty}$ robust performance.

Theorem 3.3 If the following two conditions hold:

$$
\text { 1) }\left\|M_{11}\right\|_{\mathcal{H}_{\infty}}<1 ;
$$

where $\gamma^{*} \geq 0$ is the unique solution of the equation $\gamma=G_{\gamma}(w)$. Then the system $M$ has mixed robust performance in the $\ell^{\infty} / \mathcal{H}_{\infty}$ sense for all LTV perturbations $\Delta \in \mathrm{D}_{2}$.

Proof: The first condition guarantees robust stability of the system. From Lemma 3.1, there exists a unique solution $\gamma^{*} \geq 0$ to the equation $\gamma=G_{\gamma}(w)$. Define the following sets:

$$
\begin{aligned}
& \Gamma=\left\{\gamma>0: G_{\gamma}(w) \geq \gamma\right\} \\
& \mathcal{E}=\left\{\xi:\|\xi\|_{\ell^{2}}<\|y\|_{\ell^{2}}, y=M_{11} \xi+M_{12} w\right\}
\end{aligned}
$$

From these definitions it follows that if $\xi \in \mathcal{E}$, then $\|\xi\|_{\ell^{2}}^{2} \in \Gamma$. From the monotonicity and continuity of $G_{\gamma}(w)$ we have that for all $\gamma \in \Gamma$, $\gamma \leq \gamma^{*}$. Using the monotonicity of $F_{\gamma}$ and the second assumption in the hypothesis yields:

$$
F_{\sqrt{\gamma}}(w) \leq F_{\sqrt{\gamma^{*}}}(w) \leq 1
$$

Therefore, for all $\xi \in \mathcal{E},\|z\|_{\ell^{\infty}} \leq F_{\|\xi\|_{\ell^{2}}}(w) \leq$ 1. The proof is completed by noting that since $\|\Delta\| \ell^{2} \rightarrow \ell^{2} \leq 1,\|\xi\|_{\ell^{2}}<\|y\|_{\ell^{2}}$ and therefore $\xi \in \mathcal{E}$.
Remark 1 From definition (8) and Lemma 3.1 it follows that

$$
\begin{aligned}
& F_{\sqrt{\gamma^{*}}}(w) \leq\left\|M_{22} w\right\|_{\ell \infty} \\
& +\left\|M_{21}\right\|\left\|_{\mathcal{H}_{2}}\left(1-\left\|M_{11}\right\| \|_{\mathcal{H}_{\infty}}\right)^{-1}\right\| M_{12} w \|_{\ell^{3}}
\end{aligned}
$$

Thus, by Corollary 3.2, the conditions obtained in Theorem 3.3 are less conservative than the one presented in Theorem 3.1.

Finally, we end this section by providing an upper bound on the worst case value of $\|z\|_{\ell \infty}$.

Lemma 3.3 For a given input $w$, the worst case value of the output sup $\|z\|_{\ell_{\infty}}$ is upper bounded by:

$$
\Delta \in \mathbf{D}_{\mathbf{2}}
$$

$$
\sup _{\Delta \in \mathrm{D}_{2}}\|z\|_{\ell \infty} \leq F_{\sqrt{\gamma^{*}}}(w) \leq F_{\gamma_{1}}(w)
$$

where $\gamma_{1}=\left(1-\left\|M_{11}\right\|_{\mathcal{H}_{\infty}}\right)^{-1}\left\|M_{12} w\right\|_{\ell^{2}}$.

Proof: The proof follows immediately by combining Lemma 3.1 and Theorem 3.3.

\section{Example}

The following example is taken from [19]. Consider the ACC benchmark problem, where the system has the following state-space realization:

$$
\begin{aligned}
& \left(\begin{array}{l}
\zeta \\
u \\
y
\end{array}\right)=P\left(\begin{array}{l}
v \\
w \\
u
\end{array}\right) \\
& P=\left[\begin{array}{cccc|ccc}
0 & 0 & 1 & 0 & 0 & 0 & 0 \\
0 & 0 & 0 & 1 & 0 & 0 & 0 \\
-1.25 & 1.25 & 0 & 0 & 1 & 0 & 1 \\
1.25 & -1.25 & 0 & 0 & -1 & 1 & 0 \\
\hline 1 & -1 & 0 & 0 & 0 & 0 & 0 \\
0 & 0 & 0 & 0 & 0 & 0 & 1 \\
0 & 1 & 0 & 0 & 0 & 0 & 0
\end{array}\right]
\end{aligned}
$$

In [19], this system was discretized using sample and hold elements at the inputs and outputs, with sampling time of 0.1 seconds, and an $l_{\infty} / \mathcal{H}_{\infty}$ controller was designed to achieve $\left\|T_{\xi v}\right\|_{\mathcal{H}_{\infty}} \sim \leq \frac{4}{3}$, and the control action $|u|$, in response to an impulse disturbance, $\sim \leq 1$. This controller has the following state-space realization:

$$
K=\left[\begin{array}{cc|c}
-1.7404 & -0.7769 & 0.9975 \\
0.9950 & 0 & 0 \\
\hline-1.1347 & 1.0044 & 4.1150
\end{array}\right]
$$


The closed-loop system, with the uncertainty 'pulled out', is shown in Figure 2. Suppose the

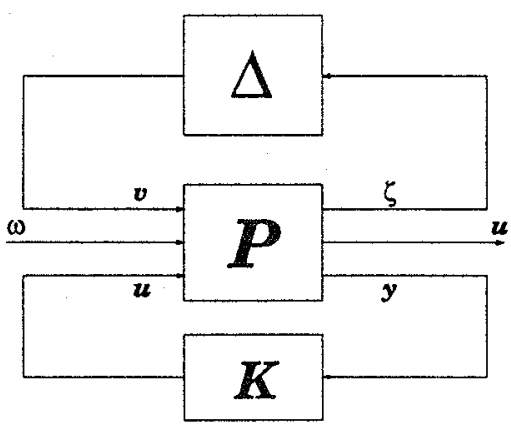

Figure 2: The standard form

LTV perturbations are limited to $\frac{1}{0.645} \Delta \in \mathrm{D}_{\mathbf{2}}$. It can be shown that

$$
\begin{aligned}
& \mu_{\Delta}\left(\mathcal{F}_{l}(P, K)\right)=3.947 \\
& \rho\left(\begin{array}{ll}
\left\|T_{\zeta v}\right\|_{l_{2}} & \left\|T_{\zeta w}\right\|_{l_{1}} \\
\left\|T_{u v}\right\|_{l_{1}} & \left\|T_{u v}\right\|_{l_{1}}
\end{array}\right)=6.821
\end{aligned}
$$

Thus, both $\mu$-analysis and $l_{1}$ theory indicate that this system doesn't have robust performance against worst-case inputs. However, by applying the proposed analysis for fixed inputs ( $l_{\infty} / \mathcal{H}_{\infty}$ robustness), we obtain that $\gamma_{1}=2.070$ and

$$
\begin{aligned}
& \rho\left(\begin{array}{ll}
\left\|T_{\zeta v}\right\|_{\mathcal{H}_{\infty}} & \left\|T_{\zeta v}\right\|_{\ell^{3}} \\
\left\|T_{u v}\right\|_{\mathcal{H}_{2}} & \left\|T_{u w}\right\|_{\ell^{\infty}}
\end{array}\right)=1.002 \\
& F_{\gamma_{1}}\left(T_{u v}, T_{u, w}\right)=0.9837<1
\end{aligned}
$$

Therefore, we conclude that for an impulse input $w$, the closed-loop system achieves mixed robust performance against $\|\Delta\|_{\ell^{2} \rightarrow \ell^{2}} \leq$ 0.645 . This is consistent with numerical results when $\Delta \in(-0.645,0.645)$, as shown in Figure 3.

\section{Conclusions and directions for further research}

In this paper we consider the problem of analyzing robust performance (in the $\ell^{\infty}$ sense) for systems subject to $\ell^{2} \rightarrow \ell^{2}$ norm bounded perturbations. This problem can be considered as a natural extension of the mixed $\ell^{\infty} / \mathcal{H}_{\infty}$, nominal performance/robust stability type problem introduced in [15], and it is relevant for cases where some of the performance specifications are given in terms of the response to a fixed, given signal.
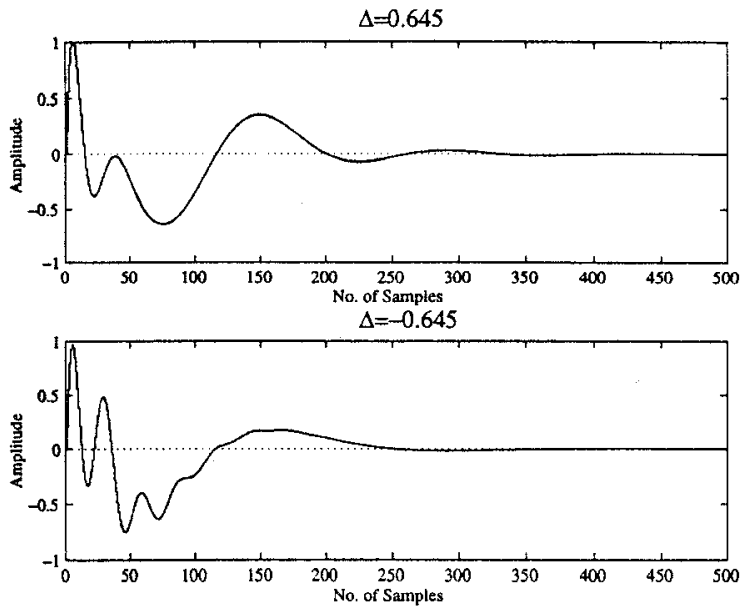

Figure 3: Impulse responses, different $\Delta$ 's

The main results of the paper provide sufficient conditions guaranteeing robust performance. One of these conditions is given in terms of the spectral radius of a matrix involving different induced norms and can be easily checked. The second condition reduces conservatism at the price of more involved computations.

These results are illustrated with a simple example taken from the literature. This example shows that although the proposed conditions are only sufficient, they provide results that are less conservative than those obtained via a worst-case input analysis, such as $\mu$-synthesis or $\ell^{1}$-theory.

The results provided here are a first step towards the goal of synthesizing multiobjective robust controllers. These controllers could be obtained for instance by combining the spectralradius based condition with standard similarity scaling and some of the techniques available for optimizing nominal performance. Research in this direction is currently being pursued.

\section{References}

[1] Franco Blanchini, Stefano Miani and Mario Sznaier, "Step Response Bounds for Time-Varying Uncertain Systems with Bounded Disturbances," Proceedings of the $34^{\text {th }}$ IEEE Conf. on Decision and Contr., New Orleans, December 1995, pp. 3188-3193.

[2] Dennis S. Bernstein and Wassim H. Haddad. LQG control with an $\mathcal{H}_{\infty}$ performance 
bound: a Riccati equation approach. IEEE Transactions on Automatic Control, 34:293305, 1989.

[3] Munther A. Dahleh and Ignacio J. DiazBobillo. Control of Uncertain Systems, A Linear Programming Approach. Prentice Hall, Englewood Cliffs, New Jersy, 1995.

[4] Nicola Elia, Peter M. Young, and Munther A. Dahleh. Robust performance for fixed inputs. In Proceedings of the 33rd IEEE Conference on Decision and Control, pages 2690-2695, 1994.

[5] Nicola Elia, Peter M. Young, and Munther A. Dahleh. Robust performance for both fixed and worst case inputs. In Proceedings of the 34th IEEE Conference on Decision and Control, pages 3170-3175, 1995.

[6] Roger A. Horn and Charles A. Johnson. Matrix Analysis. Cambridge University Press, New York, 1985.

[7] Isaac Kaminer, Pramod P. Khargonekar, and Mario A. Rotea. Mixed $\mathcal{H}_{2} / \mathcal{H}_{\infty}$ control for discrete time systems via convex optimization. Automatica, 29:57-70, 1993.

[8] Mustafa H. Khammash. Robust steady state tracking. IEEE Transactions on Automatic Control, 40:1872-1880, 1995.

[9] David G. Luenberger. Optimization by Vector Space Methods. John Wiley and Sons, New York, 1969.

[10] Pramod Khargonekar and Mario Rotea "Mixed $\mathcal{H}_{2} / \mathcal{H}_{\infty}$ Control: A Convex Optimization Approach," IEEE Trans. Autom. Contr., Vol 36, 7, pp. 824-837, 1991.

[11] D. Brett Ridgely, Lena S. Valavani, and Gunter Stein. Solution to the general mixed $\mathcal{H}_{2} / \mathcal{H}_{\infty}$ control problem-necessary condition for optimality. In Proceedings of the American Control Conference, pages 1348-1352, 1992.

[12] Murti V. Salapaka, Mohammed A. Dahleh, and Petros G. Voulgaris. Mixed objective control synthesis: optimal $l_{1} / \mathcal{H}_{2}$ control. In Proceedings of the American Control Conference, pages 1438-1442, 1995.

[13] Murti V. Salapaka, Mohammed A. Dahleh, Antonio Vicino, and Alberto Tesi. Nominal $\mathcal{H}_{2}$ Performance and $l_{1}$ Robust Performance. In Proceedings of the 35th IEEE Conference on Decision and Control, pages 4034-4039, 1996.

[14] Anton A. Stoorvogel. The robust $\mathcal{H}_{2}$ control problem: a worst-case design. IEEE Transactions on Automatic Control, 38:1358-1370, 1993.
[15] Mario Sznaier, "A Mixed $l^{\infty} / \mathcal{H}_{\infty}$ Optimization Approach to Robust Controller Design," SIAM Journal on Optimization and Control, 33, 4, pp. 1086-1101, July 1995.

[16] Mario

Sznaier and Franco Blanchini, " $\mathcal{L}^{\infty} / \mathcal{H}_{\infty} S u b$ optimal Controllers for Continuous-Time Systems," IEEE Trans. Autom. Contr.,40, 11, pp 1831-1840, November 1995.

[17] Mario Sznaier. Mixed $l_{1} / \mathcal{H}_{\infty}$ controllers for SISO discrete-time systems. System and Control Letters, 23:179-186, 1994.

[18] Mario Sznaier and Peter Dorato, organizers, Invited Session on Multiobjective Robust Control, session FA-02, Proceedings of the 33 IEEE CDC, Lake Buena Vista, Florida, December 1994, pp. 2672-2707.

[19] Mario Sznaier and Héctor P. Rotstein. Robust controller design for a non-colocated spring-mass system via mixed $\ell^{\infty} / \mathcal{H}_{\infty}$ optimization. International Journal of Robust and Nonlinear Control, 5:53-65, 1995.

[20] Petros G. Voulgaris. Optimal $\mathcal{H}_{2} / l_{1}$ control via duality theory. IEEE Transactions on Automatic Control, 40:1881-1888, 1995.

[21] Xin Chen and John T. Wen, "A Linear Matrix Inequality Approach to Discrete-Time Mixed $l^{1} / \mathcal{H}_{\infty}$ Control Problems," Invited Session on Multiobjective Robust Control II, Proceedings of the 34 IEEE CDC, New Orleans, La, December 13-15, 1995. 